\section{Cahiers de Narratologie}

Analyse et théorie narratives

$28 \mid 2015$

Le récit comme acte cognitif

\title{
L'école de Tel Aviv, une approche rhétorique et fonctionnaliste du récit
}

\section{Eyal Segal}

Translator. Raphaël Baroni*

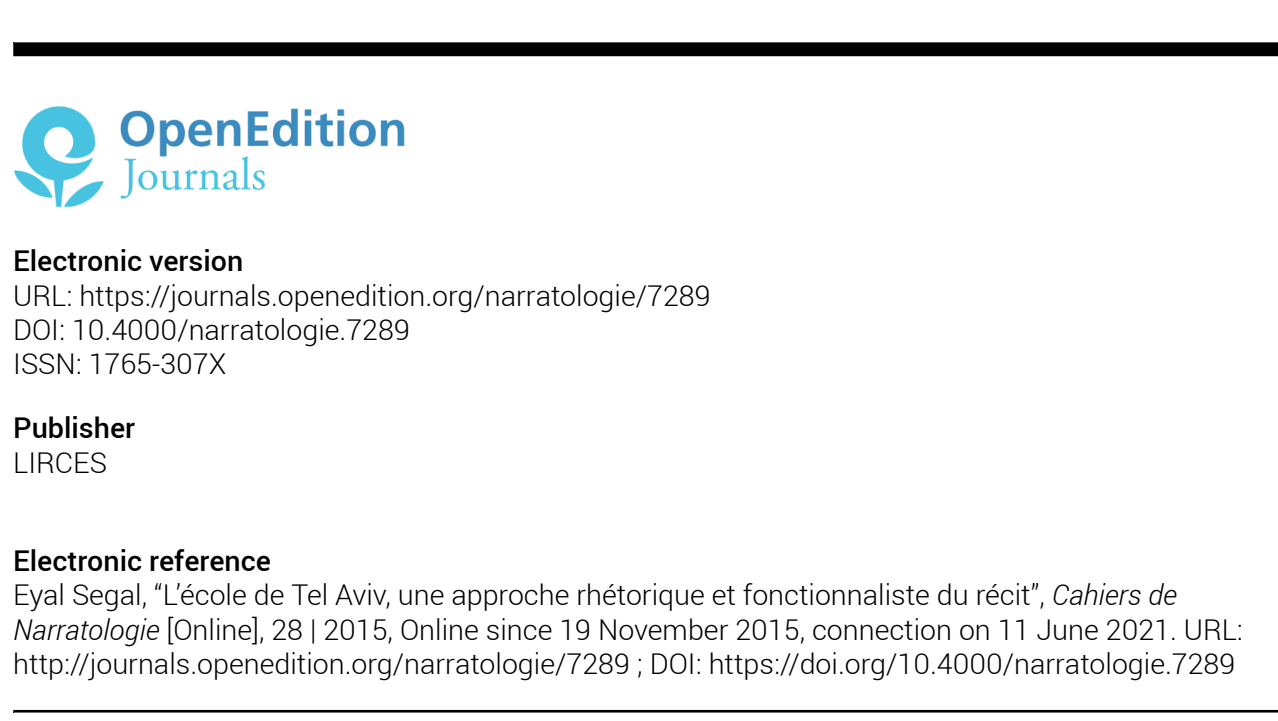

This text was automatically generated on 11 June 2021 .

Article L.111-1 du Code de la propriété intellectuelle. 


\title{
L'école de Tel Aviv, une approche rhétorique et fonctionnaliste $\mathrm{du}$ récit
}

\author{
Eyal Segal
}

Translation : Raphaël Baroni*

\section{EDITOR'S NOTE}

*Traduit de l'anglais

1 Dans cet essai, je voudrais donner un bref aperçu d'une orientation importante de la narratologie incarnée par les travaux de plusieurs théoriciens du récit israéliens qui sont tous associés au Département de poétique et de littérature comparée (DPCL) à l'Université de Tel Aviv. Ce département a été fondé par Benjamin Hrushovski au milieu des années soixante, et a commencé à avoir un impact international à partir du milieu des années soixante-dix avec la fondation en 1975 de l'Institut Porter pour la poétique et la sémiotique et le lancement de deux revues anglophones, l'éphémère PTL (Poetics and Theory of Literature) et son successeur Poetics Today (pour plus de détails historiques, voir McHale \& Segal 2015 : 196-200). Ceci étant posé, je commencerai par formuler quelques-uns des principes de base guidant l'approche du récit - et en fait de la littérature en général - propre à ce que je désignerai comme l'École de Tel Aviv.

2 La clé de voûte de cette approche est la conception que le récit est avant tout et essentiellement un acte communicatif. Dans les premiers stades de son développement, c'est-à-dire dans les années soixante et au début des années soixante-dix, cette approche recoupait de façon évidente à la fois l'ouvrage influent de Wayne Booth Rhetoric of Fiction (1961) et ce qu'on a appelé les «théories de la réception». Cette dernière orientation s'est développée simultanément en plusieurs endroits - je mentionnerai des développements aussi variés que les écrits de Stanley Fish (1980), l'article «La mort de l'auteur» (censé conduire à la «naissance du lecteur») et 
l'ouvrage S/Z de Roland Barthes (1968; 1970), les travaux de l'École de Constance (notamment Iser 1974 et Jauss 1977) et même l'influente présentation du structuralisme par Jonathan Culler dans Structuralist Poetics (1975), que je décrirais comme une réinterprétation du structuralisme à travers la perspective de la réception, point de vue qui prend forme dans le concept de "compétence littéraire ». Quoi qu'il en soit, mon but n'est pas d'établir les dates et les détails exacts de cette émergence mais de poser les fondements pour une comparaison sur une large échelle.

3 Comme toutes ces approches, les travaux produits par les membres de l'École de Tel Aviv se centrent sur l'activité du lecteur et, plus précisément, sur la dynamique du processus de la lecture. Je voudrais néanmoins préciser que, comparativement à ces modèles, l'« approche de Tel Aviv» se présente comme une conceptualisation plus équilibrée de l'acte communicatif, car elle tient compte de ses deux versants ou participants - le lecteur, pour qui le texte est construit, et l'auteur (implicite), qui a modelé le texte dans le but d'atteindre ses objectifs communicationnels. Un exemple clair de cette tendance peut être trouvé dans l'article fondateur « Le roi à travers un regard ironique » (« The King through Ironic Eyes », publié une première fois en hébreu en 1968) écrit conjointement par Meir Sternberg et Menakhem Perry, qui propose une lecture serrée de l'histoire biblique de David, Urie et Bath-Shéba (Bethsabée) dans le livre de Samuel. L'article inclut une discussion extensive sur l'activité de comblement des lacunes textuelles [gap-filling] par le lecteur - une activité que cette histoire biblique dramatise particulièrement à cause de son style très laconique, qui laisse beaucoup d'espace pour une reconstruction par le lecteur de ses significations implicites. Cependant, conjointement à l'accent mis par Sternberg et Perry sur l'activité du lecteur dans ce contexte, ils ne cessent de souligner également les différentes manières dont le texte contrôle et oriente - au moins jusqu'à un certain point - cette activité ${ }^{1}$.

point de vue, le travail réalisé par la narratologie de Tel Aviv - et en particulier par Meir Sternberg - peut être qualifié dans les grandes lignes comme le prolongement d'une orientation rhétorique telle que la concevait Wayne Booth. Cependant, l'obsession de Booth pour la question du jugement éthique le conduit à se focaliser sur la construction du "point de vue", à l'exclusion de pratiquement tous les autres aspects de la narrativité. À l'inverse, la "rhétorique " telle qu'elle est pratiquée par l'École de Tel Aviv est beaucoup plus ouverte et inclut, en principe, tout ce qui est communiqué par le texte et ce qui influence (ou est perçu par) le lecteur.

5 Cette orientation rhétorique est solidaire d'une approche fonctionnaliste. Au lieu de simplement décrire et classifier des formes, la narratologie de Tel Aviv explore leurs buts et motivations, guidée en cela par le « Principe de Protée » [«Proteus Principle»] tel qu'il a été formulé par Meir Sternberg. Selon ce principe, il n'y a pas de "forfaits » [package deals] dans le récit (ou dans la communication en général) et pour cette raison, les rapports entre les traits ou les formes apparentes et leurs effets ou fonctions sont variables. Dans différents contextes, les mêmes formes pourraient remplir des fonctions différentes et une même fonction peut être remplie (ou un même effet peut être créé) par différentes formes.

6 Dans ce qui va suivre, je voudrais présenter un échantillon du travail réalisé par la narratologie de Tel Aviv, de manière à fournir, en relation avec différents thèmes, quelques mises en oeuvre concrètes de ce que j'ai évoqué jusqu'ici. Je commencerai avec l'étude par Tamar Yacobi de ce qui est habituellement considéré comme une 
question relative au "point de vue » : la narration non-fiable. Ensuite, je passerai aux travaux de Meir Sternberg qui s'attachent à une question que l'on associe généralement l'intrigue : la définition du récit et de la narrativité. Enfin, je voudrais brièvement présenter les bases de ma propre étude de la clôture narrative, qui peut être considérée comme une application et une extension du concept de narrativité développé par Sternberg et donc - c'est du moins mon espoir - comme un exemple de la productivité de son approche.

7 La conception que se fait Tamar Yacobi $(1981,1987,2001,2005)$ de la narration nonfiable diffère fondamentalement des autres approches de ce phénomène (en commençant par celle de Wayne Booth) car elle ne la considère pas comme un trait de caractère fixe qui serait attaché au portrait d'un narrateur, mais plutôt comme une hypothèse formulée par le lecteur de manière à résoudre une série de problèmes textuels. En tant que telle, la postulation de la non-fiabilité est toujours un acte interprétatif et hypothétique. Par un tel acte, nous (lecteurs) mettons en branle un mécanisme intégratif qui amène des éléments discordants à former une structure cohérente que nous attribuons à la singularité du discoureur ou de l'observateur à travers la médiations duquel le monde est représenté. Les déficiences du médiateur c'est-à-dire son incompétence, son manque de sincérité, son ignorance, son manque de discernement - que l'on parvient à expliquer par ce biais, sont alors utilisées pour accomplir différentes visées: rhétorique (notamment la production d'ironie), psychologique, thématique, etc. Et par une hypothèse de congruité inverse, nous pouvons déduire et expliquer ce que serait une narration fiable.

8 Ce mécanisme d'intégration perspectivale [perspectival integration] n'est, en fait, qu'une possibilité parmi d'autres types de mécanismes (ou de logiques) possibles de résolution, qui se trouvent en compétition et qui peuvent être activés par le lecteur. Les quatre autres mécanismes sont les suivants :

9 - Le mécanisme génétique qui attribue les étrangetés et les incohérences de la fiction à la production du texte et à ses circonstances. Pour dire les choses brutalement, cela «met la faute sur l'auteur ». Ce mécanisme est en fait, dans certains cas, assez proche du mécanisme "perspectival» en ce que lui aussi fait porter la responsabilité des étrangetés textuelles sur quelqu'un qui est associé à l'histoire racontée. Il y a cependant une différence dans l'identité de la personne responsable. Une explication génétique impute souvent à l'auteur une certaine perte de contrôle sur le texte, alors que l'hypothèse d'un narrateur faillible conduit à attribuer à l'auteur, en tant que communicateur qui se cache derrière la scène, une maîtrise ironique.

- Le mécanisme générique qui fait appel à un certain modèle de réalité (ou à une simplification de cette dernière) qui est encodé en fonction de conventions génériques ; il s'agit par exemple de la liberté causale propre à la comédie, qui s'oppose à une logique plus stricte qui serait typique de l'intrigue tragique.

- Le mécanisme fonctionnel qui impose un ordre sur la déviance en postulant que certains buts la requièrent ou la justifient.

- Le mécanisme existentiel qui rattache les incongruités à la dimension « objective » du monde fictif et à sa logique interne - notamment les canons de la probabilité, qui s'écartent sensiblement de ceux propres à la réalité.

10 Je voudrais insister sur quelques-uns des bénéfices que l'on peut tirer de l'approche de Yacobi. Premièrement, dans l'idée - articulée plus haut selon le « Principe de Protée »de briser les liens automatiques entre forme et fonction, cette approche évite certaines 
assimilations fâcheuses, qui prévalent encore dans la caractérisation du narrateur. Parmi elles, celle qui est la plus courante concerne la postulation d'un rapport automatique entre, d'une part, la non fiabilité et la narration homodiégétique ou à la première personne et, d'autre part, entre la fiabilité et la narration hétérodiégétique ou à la troisième personne (ce point est développé dans Yacobi 2001).

De plus, l'approche de Yacobi place la question de la non fiabilité dans un contexte plus large que d'habitude, ce qui lui permet, entre autres choses, de décrire et de mettre en relation des postures interprétatives divergentes concernant un même texte, en les abordant sous l'angle d'une théorie de l'interprétation à large spectre qui reste malgré tout spécifiquement orientée vers la narration elle-même. On peut trouver un exemple de ce type, sur une grande échelle, dans une étude de cas portant sur la réception de $L a$ Sonate à Kreutzer de Tolstoï (Yacobi 2005), ce texte ayant généré une riche polémique en ce qui concerne la question de la fiabilité du narrateur. Pour rester bref, je voudrais démontrer l'intérêt de cette approche en me fondant sur une autre controverse interprétative fameuse, concernant le livre de Henry James Le Tour d'écrou. La controverse se concentre sur la question de savoir si les fantômes qui apparaissent dans l'histoire de la gouvernante sont réels ou s'ils sont le produit de l'imagination névrotique de la narratrice. Dans les termes du modèle de Yacobi, il s'agit d'un cas évident de conflit entre le mécanisme d'intégration perspectivale, qui explique (ou, pour les pro-apparitionnistes, qui feint d'expliquer) que les fantômes sont le produit d'une narration non fiable, et le mécanisme existentiel, qui attribue aux fantômes une existence objective dans le monde représenté par le texte. Ce dernier mécanisme est étayé, dans ce cas précis, par le mécanisme générique de l'« histoire de fantôme » et par le canon spécifique de probabilité qui lui est associé.

Quoi qu'il en soit, la controverse entourant le récit de Henry James ne s'arrête pas là, étant donné qu'à un certain stade de son développement, une autre hypothèse a acquis un statut dominant, statut dont je pense qu'elle jouit encore aujourd'hui. Cette hypothèse maintient que le texte est systématiquement ambigu, et que le débat entre les interprétations opposées que j'ai mentionnées ci-dessus n'est en fait que le reflet de cette ambiguïté structurale qui est au principe de son organisation. Ainsi, cette " métahypothèse " (pour le dire ainsi), motive le statut problématique des fantômes par un autre mécanisme d'intégration, à savoir le mécanisme fonctionnel, qui place l'ambiguïté comme la finalité de l'œuvre².

13 Je voudrais maintenant me tourner vers les travaux de Meir Sternberg, qui portent sur la définition et la compréhension du récit, et en particulier de la «narrativité »- de ce qui constitue l'essence d'un récit ${ }^{3}$.

14 Contrairement à la plupart des approches narratologiques, celle de Sternberg définit l'essence du récit non pas en termes mimétiques, dans l'action représentée ou narrée, mais plutôt en termes rhétoriques et communicationnels, relatifs à l'intérêt du récit. Cet intérêt est suscité chez le lecteur par la création de lacunes informationnelles concernant n'importe quel aspect du monde représenté - que ce soit un événement, un motif d'agir, un trait de caractère, une relation, un point de vue, une image de la société ou même un modèle complet de réalité. Fondamentalement, ces lacunes résultent de l'interaction entre deux temporalités : celle de la séquence des événements représentés (semblable à la vie) et celle de leur dévoilement artistique, à travers la séquence du raconter/lire; ou, en abrégé, le mimétique et le textuel. Sternberg définit trois types d'intérêts narratifs fondamentaux, en fonction des combinaisons possibles entre ces 
deux niveaux séquentiels (voir tableau infra). On peut définir les différentes entrées de ce tableau en fonction de deux types de questions : 1. Est-ce que l'information cachée au lecteur appartient au futur ou au passé (les deux perspectives temporelles étant reliées avec ce qui est perçu, à un point donné de la séquence textuelle, comme le "présent» du récit)? 2. Le lecteur est-il conscient qu'une information lui est dissimulée? (en d'autres termes, sait-il ou elle qu'il ou elle ne sait pas quelque chose ?) La conscience de ne pas savoir quelque chose crée une attente portant sur l'information manquante, qui se transforme soit en suspense - si la lacune est située dans le futur du récit -, soit en curiosité - si la lacune porte sur le passé.

Néanmoins, si le lecteur n'est pas au courant qu'une lacune existe, cette dernière deviendra effective ou perceptible seulement au moment ou elle sera révélée de manière impromptue, créant ainsi de la surprise. Nous voyons que seulement trois des quatre cases ci-dessous sont remplies, ce qui révèle une asymétrie de base entre le passé et le futur : il est évident que l'on ne peut pas être surpris par quelque chose qui n'est pas encore arrivé.

Les trois types principaux de l'intérêt du récit

\begin{tabular}{|l|l|l|}
\hline Futur & Passé & \\
\hline Suspense (Prospection) & Curiosité (Rétrospection) & Attente (Connaissance d'une lacune) \\
\hline $\mathrm{X}$ & Surprise (Recognition) & Absence d'attente (Ignorance de la lacune) \\
\hline
\end{tabular}

Dans ce contexte, il peut être utile de tracer une distinction supplémentaire entre deux types de surprise. Quand l'existence d'une lacune dans ce qui était précédemment considéré comme un passé pleinement révélé est découverte, la lacune elle-même peut être comblée immédiatement, mais elle peut aussi rester ouverte (ou pour le moins elle peut ne pas être comblée sans ambiguïté), ce qui créée par conséquent de la curiosité. Par exemple, la découverte soudaine de l'existence d'un secret obscur dans le passé d'un personnage peut survenir pendant la révélation du secret lui-même, ou laisser la nature de ce secret inconnu. Dans les deux cas, il y aura de la surprise, mais ce n'est que dans le second qu'elle laissera ou générera une " trace » de curiosité.

En somme, ces trois types principaux d'intérêt narratif mettent en lumière des fonctionnements qui distinguent la narrativité de toute autre forme de discours, et un texte dans lequel de tels traits dominent (isolément ou en combinaison) peut être décrits comme un récit.

Je voudrais montrer brièvement la différence qui existe entre une approche du récit de ce genre, à la fois rhétorique et fonctionnelle, qui met l'accent sur les mécanismes de l'intérêt narratif, et une autre approche narratologique très influente, celle de Gérard Genette, qui se fonde sur une taxinomie de formes. Je me concentrerai en particulier sur le phénomène que Genette appelle la "prolepse ». Au début de son chapitre sur l'«Ordre» dans Discours du récit (1972), Genette définit la prolepse comme une "manœuvre narrative consistant à narrer ou évoquer d'avance un événement ultérieur » (p. 82). Quand il en vient à analyser cette figure dans le détail, il met en évidence des cas où des événements appartenant au futur du récit sont racontés de manière anticipée par rapport à leur situation « naturelle » dans l'ordre chronologique, 
que ce soit de manière détaillée, ou au contraire brièvement, comme des " annonces " ou " amorces "; il subdivise et classifie les cas de figure selon diverses sous-catégories : suivant que la prolepse est « externe » ou « interne » à l'histoire, suivant sa portée, son extension, etc.

Il s'agit d'une procédure d'analyse très naturelle et qui n'est pas problématique en ellemême. Néanmoins, quand on aborde la « prolepse » non en tant que forme textuelle ou en tant qu'opération spécifique (l'opération de faire une "annonce » anticipée) mais plutôt sous l'angle d'une approche fonctionnaliste - qui consiste à analyser la fonction proleptique consistant à révéler des éléments futurs de l'histoire au lecteur - l'image change radicalement. Il devient alors clair que le phénomène "proleptique » dont s'occupe Genette constitue seulement une partie - et pas nécessairement la plus importante - d'un spectre beaucoup plus large de moyens qui ont tous en commun une même visée : canaliser les anticipations du lecteur à travers le futur du récit en communiquant certaines informations à ce sujet. Parmi ces moyens, on peut mentionner par exemple la manière dont opère la familiarité du lecteur avec des conventions génériques, moyen souvent très efficace pour orienter le futur (que l'on songe aux systèmes codifiés très élaborés du genre policier classique) sans qu'il soit nécessaire d'inscrire explicitement la prolepse dans le texte. Pour donner un autre exemple, lié à une poétique plus spécifique, on peut aussi mentionner ce que Sternberg appelle "l'épithète proleptique " qui opère dans le récit biblique. Dans la poétique biblique, ainsi que le montre Sternberg (1985), il n'y a jamais d'épithète formelle renvoyant à l'attribut d'un personnage au début d'une narration qui soit redondante, ou même qui demeure statique, quand on la met en relation avec le développement ultérieur de l'histoire. Au contraire, cet attribut s'insère dans l'action et joue un rôle essentiel dans l'intrigue, acquérant ainsi une fonction proleptique implicite.

Par ailleurs, on peut noter qu'une " annonce " explicite est un trait textuel qui peut remplir différentes fonctions quand on la met en rapport avec la manipulation de l'intérêt du lecteur: de manière évidente, l'annonce peut être utilisée soit pour neutraliser l'intérêt du lecteur en divulguant de manière anticipée et complète un développement ultérieur ou, à l'inverse, elle peut renforcer l'intérêt d'un tel développement en communiquant une information limitée et suggestive, ce qui creusera l'appétit narratif du lecteur au lieu de le combler.

Ce genre de recherche qui vise à établir des corrélations fonctionnelles se situe en fait au-delà du champ d'investigation de l'approche de Genette; aussi, quand à la fin de Discours du récit il déclare qu'il n'avait pas l'intention de produire "une "synthèse" finale où se rejoindraient et se justifieraient les uns les autres tous les traits caractéristiques du récit proustien» (1972: 271), c'est qu'en fait il ne peut tout simplement pas y arriver, en tout cas pas à l'intérieur des frontières de sa narratologie, qui est atomiste par nature.

24 Pour terminer, je voudrais présenter les bases de ma propre étude de la clôture narrative (Segal 2007, 2010), qui tente de réaliser une synthèse entre le modèle narratologique de Sternberg et la conception générale de la clôture telle qu'elle est présentée par Barbara Herrnstein Smith dans son étude classique Poetic Closure (1968).

Je commencerai par clarifier ce que j'appelle une "clôture » en différenciant cette notion de la «fin». Quand nous disons qu'un texte narratif est «fini », on peut simplement vouloir dire que la narration a atteint son point final, ce qui signifie que nous nous référons à un phénomène incontournable (et même évident), étant donné 
que tout récit doit se terminer quelque part. Sur un autre plan, on peut se référer au sens d'une fin - c'est le phénomène auquel se réfère précisément le titre du fameux livre de Frank Kermode The Sense of an Ending (1967) - ce qui signifie que l'on ne désigne pas le point final du texte en lui-même, mais plutôt un certain effet ou une qualité perceptible créée par le texte, qui peut être une résolution stable, une finalité ou une conclusion - «clinch » pour utiliser la formulation de Barbara Herrnstein Smith. Dans un tel cas, le terme "clôture " serait plus approprié. Ce n'est certainement pas un phénomène inévitable, mais plutôt l'un de ceux dont la création nécessite l'usage de stratégies textuelles assez complexes et sophistiquées, que certains textes peuvent ne pas arriver à produire, ou encore qu'ils peuvent intentionnellement éviter de créer. Dans de tels cas, on pourra parler d'" ouverture ", un concept qui se rattache encore à la même métaphore ou au même champ sémantique.

Un des avantages principaux du modèle de Sternberg tient à l'orientation rhétorique et communicationnelle de sa définition de la narrativité, et ce sont dans ces termes que je définis le concept de clôture, tout en suivant l'approche de Smith. Par conséquent, le lien entre les deux théories peut être établi assez naturellement. De manière à le démontrer, je commenterai un passage clé de l'étude de Smith dans lequel elle définit la clôture en relation avec la structure dynamique du texte perçu comme un tout :

Il sera utile de considérer la structure d'un poème comme reposant sur les principes à travers lesquels il a été généré ou en fonction desquels tel élément suit un autre. La description de la structure d'un poème devient alors la réponse à la question «Qu'est-ce qui fait qu'il se poursuit?» ... Cela permet de poser une question corollaire telle que "Qu'est-ce qui l'empêche de se poursuivre? » Ce qui suggère immédiatement qu'il existe une relation étroite entre la structure poétique et la clôture ${ }^{4}$.

Or, si la question essentielle à laquelle il s'agit de répondre quand on veut décrire la structure d'un texte est «Qu'est-ce qui fait qu'il se poursuit? » alors la conception de la narrativité articulée par Meir Sternberg pourrait être en mesure de fournir une réponse fondamentale pour les textes narratifs, à savoir: l'intérêt narratif, dans ses trois modalités de base, est ce qui fait que le récit se poursuit. La question complémentaire mentionnée ensuite par Smith, qui nous oriente vers la compréhension de la manière dont la clôture est créée (ou non), est "Qu'est-ce qui empêche le texte de se poursuivre? » et dans les termes de Sternberg, la réponse naturelle serait: la cessation ou l'achèvement de l'intérêt narratif. Pour donner un exemple simple : le type dominant de l'intérêt narratif dans le roman policier classique est la curiosité, qui dérive elle-même du mystère du crime qui est commis avant le début de l'enquête (et donc qui appartient au passé du récit). Cet intérêt se combine néanmoins avec un suspense portant sur l'issue de l'enquête, qui appartient au futur du récit. L'aboutissement de l'enquête, en apportant la solution du crime mystérieux, résout (ou «abolit») simultanément, sur une large échelle, à la fois la curiosité et le suspense.

À l'inverse, l'« ouverture » narrative est le résultat de lacunes significatives relative au monde représenté, lacunes qui demeurent en état d'ouverture (et qui ne seront pas définitivement comblées, même à la fin); en d'autres termes, elle est engendrée par des lacunes durables. Il peut s'agir aussi bien de lacunes engendrant du suspense, quand elles se rapportent au futur du récit, que de lacunes engendrant de la curiosité, quand elles se réfèrent au passé. Parmi les trois types de base définissant l'intérêt narratif, l'ouverture ne peut être décrite qu'en termes de suspense ou de curiosité, étant donné 
que cette étape nécessite que le lecteur ait conscience qu'une information importante lui manque. L'ouverture narrative ne peut pas se manifester à travers la surprise, bien qu'elle puisse certainement être causée par elle ou qu'elle puisse lui succéder - par exemple quand nous sommes confrontés à une surprise qui révèle l'existence d'une lacune sans la combler, ce qui laisse ou génère une «trace " sous forme d'attente de plus amples informations.

Il me faut préciser que ce que j'ai défini n'est pas, à strictement parler, la (non)clôture du récit, mais plutôt celle de la narrativité. Cela signifie que je ne prétends pas qu'une explication dans les termes de l'aboutissement (ou du non aboutissement) de l'intérêt narratif se réfère à la clôture (ou à l'ouverture) de tout ce qui constitue un récit. Par exemple, le texte peut faire usage de toutes sortes de dispositifs linguistiques et stylistiques qui, en eux-mêmes, n'ont aucun rapport direct avec la narrativité. Je soutiens néanmoins que, pour autant que la narrativité soit dominante dans un texte, l'explication de la clôture dans les termes d'une manipulation de l'intérêt narratif sera toujours d'une importance cruciale. Et cela nous permet aussi de mieux comprendre comment les éléments narratifs essentiels interagissent avec d'autres forces discursives importantes qui travaillent à (ou contre) l'achèvement de la dynamique du récit.

Je crois que cette définition des conditions déterminant la clôture ou l'ouverture narrative met en évidence le fait que la distinction entre fin "ouverte» ou fin « fermée » est en fait très grossière dans sa forme de base, et qu'elle ne devrait pas être regardée comme (ou réduite à) une simple dichotomie. Au contraire, la distinction devrait être affinée et placée sur une échelle possédant une gradation fine (et multidimensionnelle) tenant compte de divers aspects de la narrativité, tels que les différentes lignes d'intérêt $d u$ récit qui sont développées par le texte, leurs interrelations et leurs hiérarchisations relatives, il faudrait encore se demander jusqu'à quel point chacune de ces lignes débouche sur une clôture et, en définitif, considérer l'effet combiné de tous ces facteurs.

31 Dans le cadre de cet article, il m'est impossible d'aller au-delà de ces quelques généralités, mais je voudrais malgré tout mentionner le fait que mes travaux examinent dans le détail les structures de l'intérêt narratif propres à trois corpus différents, dont je considère que la forme de la clôture présentent un aspect singulier. En premier lieu, $j$ 'analyse des récits qui se rattachent au genre du roman policier (cas que j'ai déjà mentionné) et qui représentent le paradigme d'une clôture forte ; ensuite je me suis intéressé au roman picaresque et aux œuvres de Kafka. Ces deux derniers cas sont analysés comme des exemples où la structure de base qui défini leur intérêt narratif cause des problèmes fondamentaux au niveau de la création d'une clôture.

Pour terminer sur une note personnelle, au début de cet article, j'ai mentionné un article, «Le roi à travers un regard ironique », qui est l'étude la plus ancienne de l'Ecole de Tel Aviv figurant dans ma bibliographie. Avoir lu cet article au lycée a joué un rôle important dans ma décision d'étudier la littérature ; aussi, le fait que je sois aujourd'hui en mesure de citer une référence à mes propres travaux à côté de cet article a beaucoup d'importance à mes yeux. J'espère ne pas être trop présomptueux en affirmant que cela souligne la vitalité de l'approche du récit (et de la littérature en général) que je me suis donné pour tâche de présenter ici. 


\section{BIBLIOGRAPHY}

Une bibliographie indicative de la narratologie de l'École de Tel-Aviv :

Perry, Menakhem 1979 « Literary Dynamics : How the Order of the Text Creates Its Meaning [with an Analysis of Faulkner's “A Rose for Emily”] ", Poetics Today, 1(1-2) : 35-64, 311-61.

Perry, Menakhem, and Meir Sternberg

1986 [1968] « The King through Ironic Eyes : Biblical Narrative and the Literary Reading

Process », Poetics Today 7(2) : 275-322.

Segal, Eyal

2007 The Problem of Narrative Closure: How Stories Are (Not) Finished, PhD diss., Tel Aviv University [in Hebrew].

2010 «Closure in Detective Fiction », Poetics Today 31(2) : 153-215.

Sternberg, Meir

1978 Expositional Modes and Temporal Ordering in Fiction (Baltimore : Johns Hopkins University Press).

1985 The Poetics of Biblical Narrative : Ideological Literature and the Drama of Reading

(Bloomington : Indiana University Press).

1990 « Telling in Time (I) : Chronology and Narrative Theory », Poetics Today 11(4): 901-48.

1992 « Telling in Time (II) : Chronology, Teleology, Narrativity », Poetics Today 3(3) : 463-541.

2001 « How Narrativity Makes a Difference », Narrative 9(2) : 115-22.

2010 « Narrativity : From Objectivist to Functional Paradigm », Poetics Today 31(3) : 507-659.

Yacobi, Tamar

1981 « Fictional Reliability as a Communicative Problem », Poetics Today 2(2) : 113-126.

1987 « Narrative and Normative Pattern : On Interpreting Fiction », Journal of Literary Studies 3 :

$18-41$.

2001 « Package-Deals in Fictional Narrative : The Case of the Narrator's (Un)Reliability », Narrative 9(2) : 223-229.

2005 « Authorial Rhetoric, Narratorial (Un)Reliability, Divergent Readings : Tolstoy's Kreutzer Sonata », in A Companion to Narrative Theory, éd. James Phelan et Peter J. Rabinowitz, 108-123 (Malden, MA : Blackwell).

\section{Autres références}

Barthes, Roland1970S/Z(Paris, Seuil).1984 [1968] « La mort de l'auteur », in Le bruissement de la langue, 61-67 (Paris, Seuil).

Beidler, Peter G. 2004 « A Critical History of The Turn of the Turn of the Screw », in Henry James, the Turn of the Screw : Complete, Authoritative Text with Biographical, Historical, and Cultural Contexts, Critical History, and Essays from Contemporary Critical Perspectives, éd Peter G. Beidler (2n ed.), 189-222 (Boston : Bedford/St. Martin's).

Booth, Wayne C. 1961 The Rhetoric of Fiction (Chicago : University of Chicago Press). 
Culler, Jonathan 1975 Structuralist Poetics : Structuralism, Linguistics and the Study of Literature (Ithaca : Cornell University Press).

Fish, Stanley E. 1980 Is There a Text in this Class ?: The Authority of Interpretive Communities (Cambridge, Mass. : Harvard University Press).

Genette, Gérard1972Figure III(Paris, Seuil).

Iser, Wolfgang1976 [1974]L'Acte de lecture. Théorie de l'effet esthétique,traduit parEvelyne Sznycer, (Bruxelles, Pierre Mardaga).

Jauss, Hans R.1978 [1977]Pour une esthétique de la réception, traduit parClaude Maillard

(Paris, Gallimard).

Kermode, Frank 1967 The Sense of an Ending : Studies in the Theory of Fiction (London : Oxford University Press).

McHale, Brian, and Segal, Eyal 2015 « Small World: The Tel Aviv School of Poetics and Semiotics ", in Theoretical Schools and Circles in the Twentieth-Century Humanities : Literary Theory, History, Philosophy, édité par Marina Grishakova et Silvie Salupere, 196-215 (New York : Routledge).

Smith, Barbara H. 1968 Poetic Closure : A Study of How Poems End (Chicago : Chicago University Press).

\section{NOTES}

1. Dans ce contexte, voir aussi le sous-titre évocateur de Perry (1979): « Comment l'ordre du texte crée son sens".

2. Pour un compte-rendu détaillé de l'histoire critique que j'ai brièvement résumée, voir Beidler (2004).

3. Cette approche est élaborée en particulier dans Sternberg $(1978,1990,1992,2001,2010)$.

4. Cf. Smith $1968: 4$.

\section{ABSTRACTS}

Cet article est la traduction française d'une conférence donnée le 16 juin 2007 dans le cadre du colloque «Current Trends in Narratology » à l'Université de Fribourg (Allemagne). Il s'agit d'une présentation synthétique des théories sur le récit qui se rattachent à l'École de Tel Aviv. L'auteur souligne notamment la valeur des travaux de Tamar Yacobi, qui portent sur les narrations nonfiables, et de Meir Sternberg, dont l'originalité consiste à fonder sa définition de la narrativité sur l'intérêt narratif, qui peut prendre les formes du suspense, de la curiosité ou de la surprise. Ce courant narratologique, bien que souvent précurseur et très influent dans le monde anglo-saxon (notamment grâce à la célèbre revue Poetics Today) est encore mal connu en France du fait de l'absence de traduction de ses textes majeurs. L'article a été publié une première fois en 2007 sur le site Vox Poetica. La présente traduction a été révisée avec l'aide de l'auteur. 